\title{
Random Motion of Deuterons in $\mathrm{KD}_{2} \mathrm{PO}_{4}^{\dagger}, *$
}

\author{
V. Hugo Schmmt $\ddagger$ and Edwin A. Uehuing \\ Department of Physics, University of Washington, Seattle, Washington
}

(Received December 5, 1961)

\begin{abstract}
Magnetic resonance studies of the deuteron in $\mathrm{KD}_{2} \mathrm{PO}_{4}$ have been conducted which show the existence of deuteron jumping between and within hydrogen bonds. The experimental results help to explain electrical conductivity and ferroelectric phenomena in crystals of this type.

Pulse magnetic resonance experiments show that the lifetime $T_{X Y}$ against deuteron jumping between $X$-and $Y$-oriented hydrogen bonds is $15 \mathrm{msec}$ at $70^{\circ} \mathrm{C}$ with a jump activation energy of approximately $0.58 \mathrm{ev}$. The $c$-axis electrical conductivity of $\mathrm{KD}_{2} \mathrm{PO}_{4}$ is found to have the same activation energy, with a value of $1.16 \times 10^{-10}(\mathrm{ohm} \mathrm{cm})^{-1}$ at $25^{\circ} \mathrm{C}$.

The $\Delta m=1\left(P_{1}\right)$ and $\Delta m=2\left(P_{2}\right)$ deuteron spin-lattice relaxation transition probabilities due to $X-Y$ jumps have been calcu-
\end{abstract}

\section{INTRODUCTION}

$\mathrm{D}$ EUTERON jumps of two types are studied in this investigation of the $\mathrm{KD}_{2} \mathrm{PO}_{4}$ crystal. The deuterons (or protons, in the unsubstituted crystal) reside in hydrogen bonds which tie together linear chains of alternate $\mathrm{PO}_{4}$ tetrahedra and $\mathrm{K}$ ions. The two kinds of jumps are: (a) among the different bonds; and (b) within the individual bonds. The concept of such jumps is not new. For example, both types of jumps have been previously reported for protons in ice, ${ }^{1}$ and they have been discussed theoretically from the point of view of conductivity and dielectric relaxation phenomena by several people. ${ }^{2,3}$ The magnitudes of both the interbond and the intrabond jump probabilities are determined in the present investigation. It is shown that both probabilities are governed by an activation energy over a wide range of temperature. Then it is shown that both the magnitude and the temperature dependence of the interbond motion are in excellent agreement with conclusions which one would draw from the electrical conductivity measurements based on the assumption that the sole contribution to conductivity is the diffusion of deuterons via the interbond jumps. Finally, a possible connection is established between the measured activation energy of intrabond jumps and the steepness of the polarization curve as predicted by one of the theories of ferroelectricity in these crystals.

† Supported by the Air Force Office of Scientific Research and by grants from the National Science Foundation.

* Based on a thesis presented by V. H. S. to the Department of Physics, University of Washington, Seattle, Washington, February, 1961, in partial fulfillment of the requirement for the degree of Doctor of Philosophy.

$¥$ Present address: Department of Physics, Valparaiso University, Valparaiso, Indiana.

${ }^{1}$ M. Eigen and L. De Maeyer, Proc. Roy. Soc. (London) A247, 505 (1958).

2 N. Bjerrum, Kgl. Danske Videnskab. Selskab, Mat.-fys. Medd. 27, 1 (1951)

${ }^{3} \mathrm{~L}$. Onsager and M. Dupuis, Rendiconti della Scuola Internazionale di Fisica-Enrico Fermi (Zanidelli, Bologna, Italy, 1960). lated from the known values of $T_{X Y}$ and the electric field gradient tensors at $X$ and $Y$ deuteron sites. Their magnitudes and the dependences of these magnitudes on magnetic field, temperature, and orientation are in good agreement with experiment.

Further measurements of $P_{1}$ and $P_{2}$ separately give a component of transition probability proportional to $\exp (0.078 \mathrm{ev} / k T)$ and independent of magnetic field. The orientation dependences of $P_{1}$ and $P_{2}$ for this component indicate quadrupolar relaxation due to deuteron jumps along hydrogen bonds, with a jump time of order $10^{-11} \mathrm{sec}$ at $215^{\circ} \mathrm{K}$. The existence of intrabond jumps governed by an activation energy is shown to be consistent with the Slater theory of $\mathrm{KH}_{2} \mathrm{PO}_{4}$ as modified by Takagi.

The deuteron magnetic resonance spectrum, the components of the electric field gradient (EFG) tensor at the deuteron sites, and the nuclear relaxation times $T_{1}$ and $T_{2}$, together with the dependence of $T_{2}$ on temperature (particularly in the neighborhood of the ferroelectric transition temperature), and of $T_{1}$ on orientation of the crystal relative to the direction of the applied magnetic field, were known from a previous study. 4

The main features of the present investigation are the measurement of the interbond jump time described in Sec. II and the measurement of the separate relaxation probabilities $P_{1}$ and $P_{2}(\Delta m=1$ and 2 , respectively) leading to the discovery of the intrabond contribution as described in Sec. IV. Section III deals with the experimental verification of the interbond contribution to relaxation, thus providing a more secure basis for the evaluation of other contributions. Finally, Secs. V and VI discuss the implications of the two jump motions, insofar as electrical conductivity and the nature of the ferroelectric phase transition is concerned.

\section{MEASUREMENT OF THE JUMP TIME BETWEEN BONDS}

In the notation of $\mathrm{BU}$, two kinds of bonds are to be distinguished for the purpose of the present discussion. These are the $X$ and $Y$ bonds lying approximately in the plane perpendicular to the crystalline $c$ axis and at right angles to each other. The reason for the distinction is that these bonds experience different quadrupole splittings in a magnetic field due to the different orientations with respect to the field. We need not make the additional distinction that there are two kinds of $X$

${ }^{4}$ John L. Bjorkstam and Edwin A. Uehling, Phys. Rev. 114, 961 (1959). This paper also provides a summary of other kinds of information on this crystal which is useful in the present study; e.g., crystal structure, some properties of the hydrogen bond, etc.; also, a list of references to previous studies. Subsequent references to this paper will be to $\mathrm{BU}$. 
and two kinds of $Y$ bonds $\left(X_{+}, X_{-}\right.$and $\left.Y_{+}, Y_{-}\right)$since the relevant angular differences are small and the additional splittings are of no immediate consequence.

The present investigation began with the observation reported in $\mathrm{BU}$ that saturation of an $X$-bond line has as its consequence the saturation of a $Y$-bond line. In order to determine whether the mutual saturation effect was due to a spin flip interaction or to deuteron diffusion between $X$ - and $Y$-bond positions, the saturation effect was studied at different crystal orientations in the magnetic field. Choosing the $m=0,1$ line of an $X$ bond as the line to be saturated, the $m=0,1$ and the $m=0,-1$ lines of the $Y$ bond were observed. By varying the crystal orientation, the frequency of one or the other of the two $Y$ lines could be made to diverge most strongly from the frequency of the saturated $X$ line. It was observed that the saturated $Y$ line was always the one having the same pair of quantum numbers, independent of the frequency difference. This result was regarded as adequate proof of jumping between bonds, and the next step was the measurement of the jump time.

The measurements will show that the jump time $T_{X Y}$ (defined more precisely in Appendix I) is much less than the thermal relaxation time $T_{1}$ at room temperature and higher, and is of the order of $T_{1}$ at about $250^{\circ} \mathrm{K}$ and lower. Two methods have been devised to measure $T_{X Y}$, one applicable when $T_{X Y} \ll T_{1}$ and the other applicable when $T_{X Y} \sim T_{1}$.

In the first method (for $T_{X Y} \ll T_{1}$ ) the crystal was subjected to two $90^{\circ} \mathrm{rf}$ pulses ${ }^{5}$ separated by a time of order $T_{X Y}$, followed by a similar double pulse after about $7 \sec \left(T_{2} \ll T_{X Y} \ll 7 \sec \ll T_{1}\right)$. Thus, the measurement was completed in a time sufficiently short so that, according to the analysis of Appendix I, only one time constant concerned with the approach to equilibrium, namely $T_{X Y}$, needs to be considered.

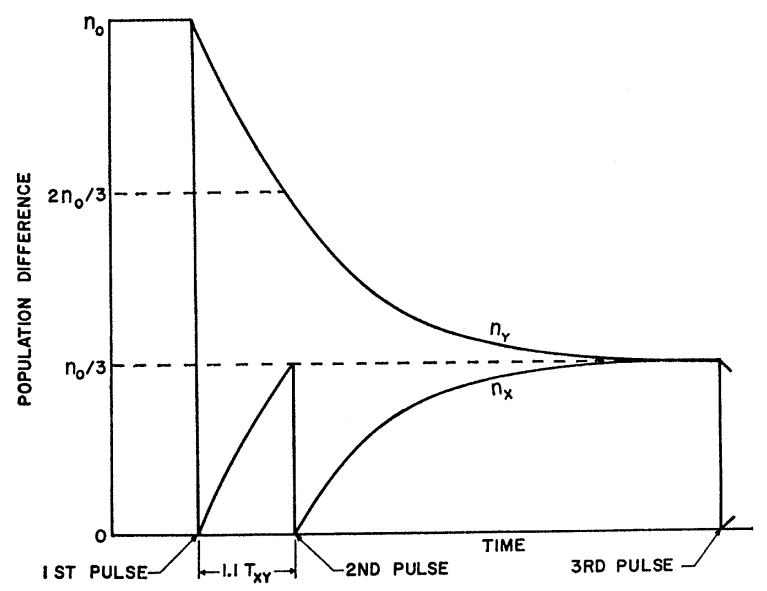

FIG. 1. Double $90^{\circ}$ pulse $T_{X Y}$ measurement method.

${ }^{5}$ E. L. Hahn, Phys. Rev. 80, 580 (1950); T. P. Das and A. K. Saha, Phys. Rev. 93, 749 (1954).

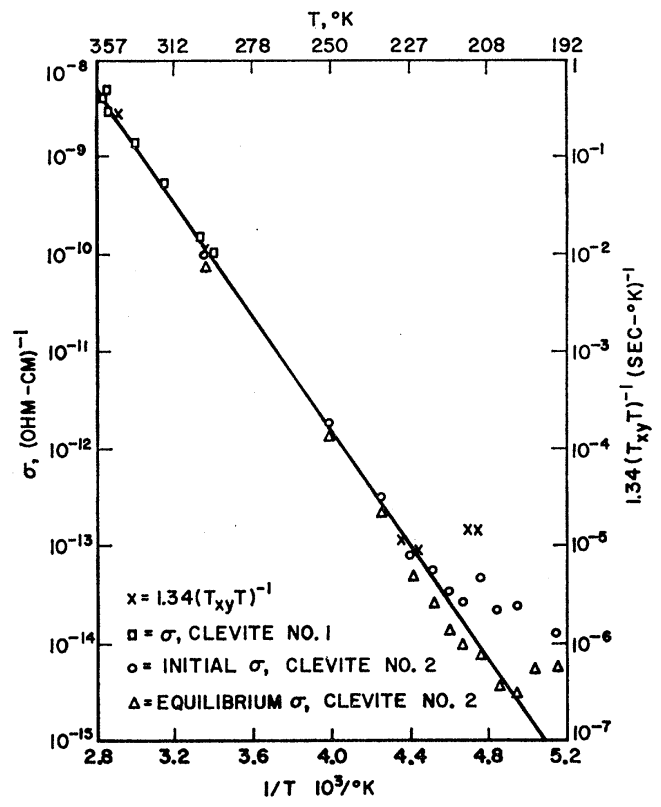

Fig. 2. Temperature dependence of electrical conductivity and $T_{X Y}$ in $\mathrm{KD}_{2} \mathrm{PO}_{4}$.

The effect of the pulse sequence on the population difference of a pair of states (e.g., states $m=m^{\prime}$ and $m^{\prime \prime}$ of an $X$-bond deuteron as determined by the pulse frequency) and of a corresponding pair of states (same $m$ values but belonging to a $Y$-bond deuteron) is then described by the free decay between pulses with time constant $T_{X Y}$. The change of population differences with time is illustrated for a particular case in Fig. 1. In order to measure $T_{X Y}$, the free induction signal from the $X$-bond deuteron is observed immediately following the application of the second and third pulses. This signal is assumed to be proportional to the population difference immediately before the application of the pulse. By varying the interval between the first and second pulses the ratio of the observed signals can be varied. As illustrated in Fig. 1 this interval can be chosen so that the two signals are of equal magnitude. Under these circumstances the response characteristics of the receiver are unimportant and need not be known. It can be shown that one obtains equal signals following the second and third pulses when the interval between the first and second pulses is equal to $T_{X Y} \ln 3$ or approximately $1.1 T_{X Y}$. Values of $T_{X Y}$ obtained by this method at room temperature and at $70^{\circ} \mathrm{C}$ are represented by two crosses in the upper left-hand corner of Fig. 2. The values are $T_{X Y}=15 \mathrm{msec}$ at $70^{\circ} \mathrm{C}$ and $400 \mathrm{msec}$ at room temperature.

The second method (useful at lower temperatures when $\left.T_{X Y} \sim T_{1}\right)$ is as follows: The crystal is oriented in the magnetic field so that the two $Y$ lines coincide. As shown in Fig. 3 this occurs when the field is perpendicular to the $Z$ axis and $\theta_{Z}=35^{\circ}$. The equilibrium 
signal height for an $X$ line is determined. Then the merged $Y$ line is saturated at a high rf level for a time long compared to $T_{X Y}$ and $T_{1}$. As quickly as possible after ending the saturation, the signal height from the $X$ line is again measured. Also, the merged $Y$-line signal is measured at times of the order of $T_{X Y}$ after ending the saturation. These signal heights are again assumed to be proportional to population differences. Figure 4 shows how the $X$ - and $Y$-population differences change as a function of time during the measurement sequence. As will now be shown, these measurements are sufficient to determine $T_{X Y}$ and $T_{1}$.

Because of the symmetry of the excitation with respect to $m=1$ and $m=-1$ spin states, the $X$ - and $Y$-spin systems are always characterized by a Boltzmann population distribution. Consequently, the time constants associated with $U_{+}$and $V_{+}$as described in Appendix I play no role. In fact, $x_{0}=y_{0}=0$; therefore, $x_{+}=-x_{-}$and $y_{+}=-y_{-}$and $U_{+}=V_{+}=0$ at all times. Thus, only Eqs. (28) and (29) in $U_{-}$and $V_{-}$are needed. In order to simplify the problem somewhat, the approximation $P_{1 X}=P_{1 Y}=P_{1}, P_{2 X}=P_{2 Y}=P_{2}$, and consequently $P_{X}=P_{Y}=P$ is made. This is strictly true at $\theta_{Z}=45^{\circ}$ since the $X$ and $Y$ bonds are then equivalent, and is only approximately true at $\theta_{Z}=35^{\circ}$. Then, the solutions of Eqs. (28) and (29) for $U_{-}$and $V_{-}$in the period following the ending of saturation are

$$
\begin{aligned}
& U_{-}=C_{1} e^{-P t}+C_{2} e^{-(P+2 P X Y) t}, \\
& V_{-}=C_{1} e^{-P t}-C_{2} e^{-(P+2 P X Y) t} .
\end{aligned}
$$

The initial condition on $V_{-}$follows from the condition of complete saturation of the merged $Y$ line; i.e., $y_{+}$ $=-n_{0}, y_{-}=+n_{0}$, and therefore $V_{-}=-2 n_{0}$ where $n_{0}$ is the equilibrium population difference of adjacent levels. The initial condition for $U_{-}$may be obtained from Eq. (28), which is valid also during the period of saturation [whereas Eq. (29) has an additional term] by taking $d U_{-} / d t=0$. Thus,

$$
U_{-}=P_{X Y} /\left(P_{X Y}+P\right) V_{-}=-2 n_{0} P_{X Y} /\left(P_{X Y}+P\right) .
$$

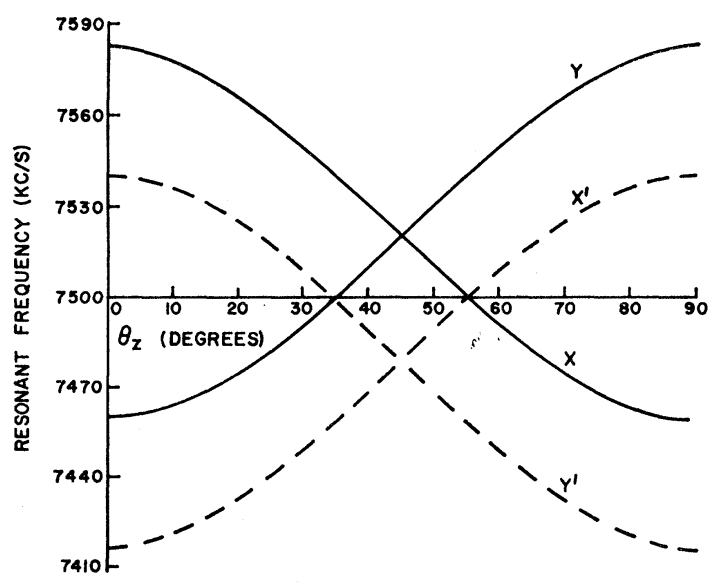

Fig. 3. D resonant frequency vs $\theta_{Z}$ in $\mathrm{KD}_{2} \mathrm{PO}_{4}$.

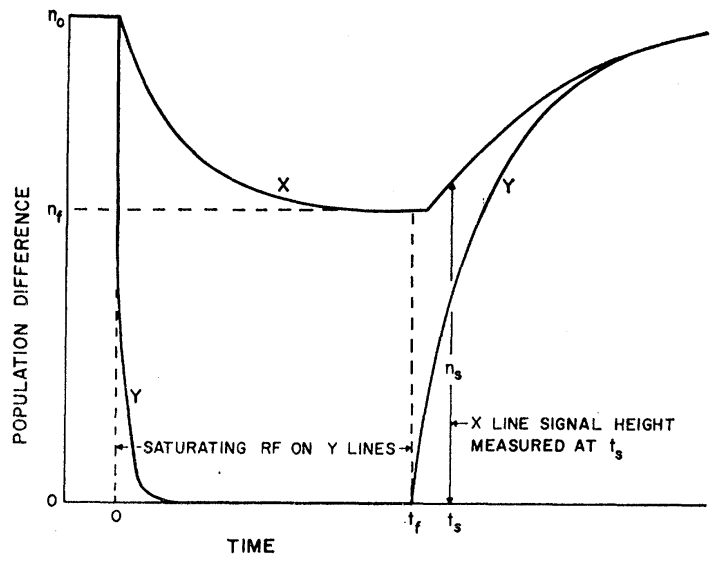

FIG. 4. $T_{X Y}$ measurement method for $T_{X Y} \sim T_{1}$.

Fixing the constants $C_{1}$ and $C_{2}$ and introducing the expressions for $U_{-}$and $V_{-}$into Eqs. (32) and (33) for the $X$ - and $Y$-population differences one obtains

$$
\begin{aligned}
& n_{x \pm}=n_{0}\left|1-\frac{P+2 P_{X Y}}{2 P+2 P_{X Y}} e^{-P t}+\frac{P}{2 P+2 P_{X Y}} e^{-\left(P+2 P_{X Y}\right) t}\right|, \\
& n_{y \pm}=n_{0}\left|1-\frac{P+2 P_{X Y}}{2 P+2 P_{X Y}} e^{-P t}-\frac{P}{2 P+2 P_{X Y}} e^{-\left(P+2 P_{X Y}\right) t}\right|
\end{aligned}
$$

Thus, one finds that the ratio of the $X$-bond signal measured immediately after ending saturation to the equilibrium signal is

$$
\frac{n_{x \pm}}{n_{0}}=\frac{P}{P+P_{X Y}}=\frac{2 T_{X Y}}{T_{1}+2 T_{X Y}} .
$$

The additional information needed to measure $T_{1}$ and $T_{X Y}$ separately is obtained by measuring $n_{y_{ \pm}} / n_{0}$ at times of the order of $T_{X Y}$ following the end of saturation.

Values of $T_{X Y}$ obtained by this method between $230^{\circ}$ and $210^{\circ} \mathrm{K}$ are given by four crosses in the lower righthand corner of Fig. 2. At $230^{\circ} \mathrm{K}, T_{X Y}$ is about $600 \mathrm{sec}$. Except for an anomalous behavior in the neighborhood of the Curie temperature, at $210^{\circ} \mathrm{K}$ in this crystal, the combined results for the jump time at high and low temperatures can be represented by the formula

$$
T_{X Y}=1.69 \times 10^{-8} T^{-1} \exp (0.58 \mathrm{ev} / k T) \mathrm{sec} .
$$

This formula takes into account also the electrical conductivity data, and will be discussed further in Sec. V. The anomalously short values of $T_{X Y}$ near the Curie temperature $T_{c}$ may be due to lower deuteron jump barriers caused by large amplitude atomic motions occurring as one of the elastic constants goes to zero at $T_{c}$.

Some description of the equipment used in these measurements may be appropriate. In the pulse measurements it was necessary to take special precautions to 
prevent rf leakage to the crystal between pulses because of the long deuteron relaxation time $\left(T_{1} \sim 10 \mathrm{~min}\right)$. A $2.5-\mathrm{Mc} / \mathrm{sec}$ crystal oscillator with a gated tripler and several gated amplifier stages was used to supply the 7.5-Mc/sec pulses to the crystal. A spectrometer head of the Weaver ${ }^{6}$ crossed coil type was used after a number of unsuccessful attempts to see the deuteron signal in the crystal using a single coil for transmitter and receiver. The receiver was a superheterodyne type with a cascode input stage. The rectified receiver output was displayed on an oscilloscope and photographed.

The low temperature measurements and all relaxation time measurements were made using a Pound$\mathrm{Knight}^{7}$ spectrometer similar to that used by BU, and like theirs modified to allow operation at a very low level. The spectrometer output went through a phase sensitive detector to a chart recorder. The magnetic field was modulated at $30 \mathrm{cps}$. All measurements made using this spectrometer employed the same general method. With the system initially in equilibrium, the line of interest was passed through using a low rf level to determine the equilibrium signal height. This signal height is the peak-to-peak height of the derivative of the absorption curve obtained by varying the magnetic field $H_{0}$ through resonance. The time of several seconds required to go through resonance was short compared to the spin system time constants, and at the low rf level used the percentage saturation resulting from going through the line was small, so that the assumption that signal height is proportional to spin level population difference is justified. After determining the equilibrium signal height, the spin system was disturbed, and after an appropriate time interval the same line was passed through again at the same low rf level to obtain the new signal height. The disturbance of the spin system consisted in some cases of an application of strong rf to saturate one of the lines (reduce the population difference for the line to zero). In other cases the magnetic field $H_{0}$ was reduced or removed.

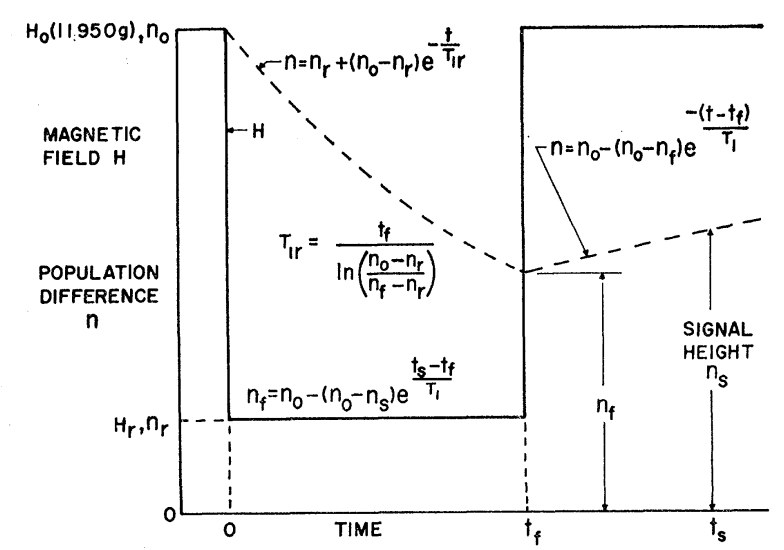

FIG. 5. $T_{1}$ measurement method for $H_{0} \leq 6000$ gauss.

${ }^{6}$ H. E. Weaver, Phys. Rev. 89, 923 (1953).

${ }^{7}$ R. V. Pound and W. D. Knight, Rev. Sci. Instr. 21, 219 (1950).

\section{RELAXATION DUE TO JUMPING BETWEEN BONDS}

The transition probabilities due to random EFG fluctuations caused by deuteron jumps between bonds are calculated in Appendix II and the results are given in Table III for two perpendicular rotations of the crystal. Since $T_{X Y}$ has been measured and $\omega=\gamma H_{0}$, no adjustable parameters appear in this calculation.

It is apparent that $P_{1}$ and $P_{2}$ due to these jumps have strong magnetic field and temperature dependence. Other mechanisms contributing to the relaxation, which will be described later, do not have these strong dependences. Consequently, it has been possible to experimentally verify the calculated relations for relaxation due to jumps between bonds, as well as to separate this contribution from the total relaxation in order to analyze other contributions.

At the high field of 11950 gauss, which is the resonant field for the spectrometer frequency of $7.8 \mathrm{Mc} / \mathrm{sec}$,

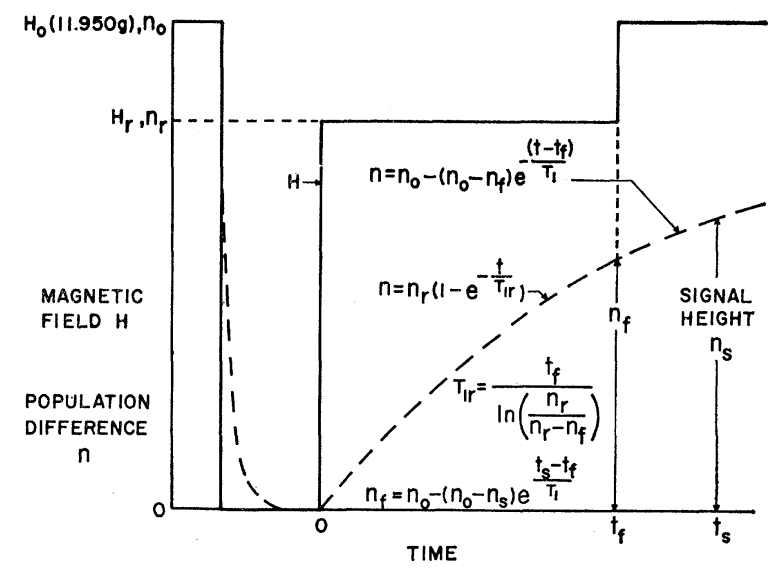

Fig. 6. $T_{1}$ measurement method for $6000 \leq H_{0} \leq 11950$ gauss.

the interbond jumps make the dominant contribution to relaxation only at temperatures considerably above room temperature. This mechanism remains dominant at lower temperatures only if the field is sufficiently reduced. In order to test this behavior and to provide the desired verification, measurements were made at several temperatures as a function of magnetic field.

The experimental sequence of Fig. (5) was used to study relaxation at fields under 6000 gauss. The field was reduced quickly from 11950 gauss to the desired value, and was left at the lower value for a time of order $T_{1 r}$, the relaxation time at the reduced field. The signal height, proportional to the population difference $n_{s}$, was obtained as soon as possible after returning to the field of 11950 gauss. A correction was applied for the time at 11950 gauss which elapsed before measuring the signal height. Because of the symmetrical effect of the field changes on spin populations, only the time constants (31) as described in Appendix I are relevant. The decay was simplified 
further because strong coupling could be assumed to exist in all cases. This was the case at high temperatures because $T_{X Y}$ is small. It was the case at low temperatures because the crystal was given a $\theta_{Z}=45^{\circ}$ orientation for which $X$ and $Y$ sites are identical. Thus, the only time constant for approach to equilibrium is $T_{1}=\left[\frac{1}{2}\left(P_{X}+P_{Y}\right)\right]^{-1}$. In Fig. (5), $T_{1 r}$ is used for $T_{1}$ to indicate that it is shorter at the reduced field.

The experimental sequence followed in order to determine $T_{1}$ at reduced fields greater than 6000 gauss is shown in Fig. (6). The populations were first equalized by turning the magnet off. This equilization was rapid because of the quadratic dependence of $T_{1}$ on field (as long as $\omega T_{X Y}>1$ ). The field was then set to the desired value for a time of order $T_{1 r}$, after which it was raised to 11950 gauss and the signal height was measured.

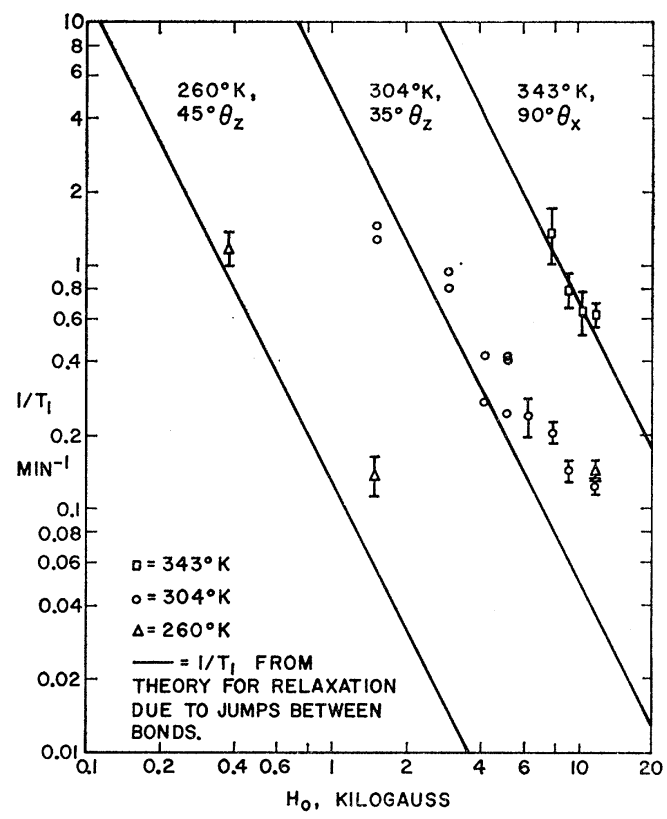

FIG. 7. Field dependence of $T_{1}$ for $\mathrm{D}$ in $\mathrm{KD}_{2} \mathrm{PO}_{4}$.

The experimental results are compared in Fig. (7) with the calculated values of $T_{1}$ due to jumping between bonds at several temperatures and specified crystal orientations. Agreement is good at high temperature and low fields where this mechanism dominates.

Further verification, in particular the angular dependence, and a more detailed analysis of the relaxation time measurements at 11950 gauss, will be deferred until after the other contributions to the relaxation have been discussed in the next section together with the measurements of $P_{1}$ and $P_{2}$ separately. It will then be shown that the predicted dependence of $P_{1}$ and $P_{2}$ on crystal orientation as well as the magnitudes are in good agreement with the experimental results.

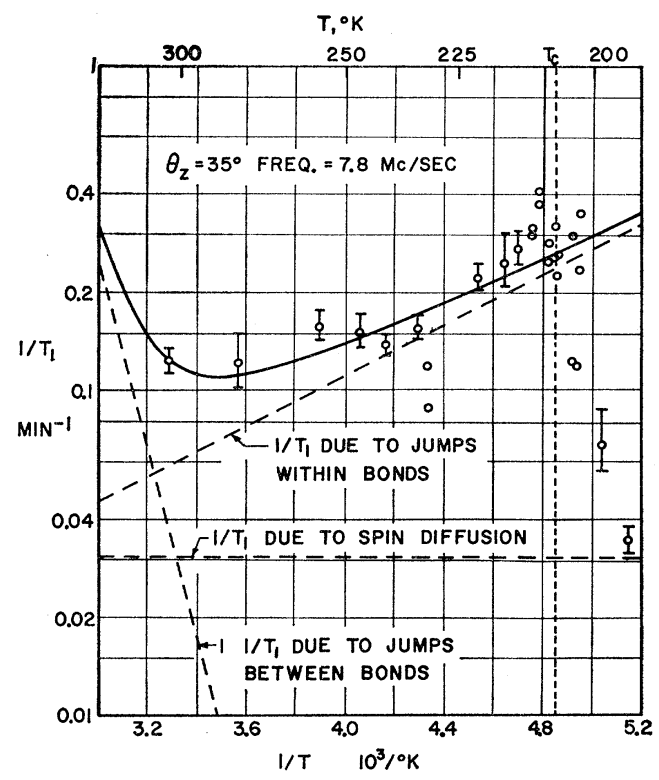

Fig. 8. Relaxation mechanisms for $\mathrm{D}$ in $\mathrm{KD}_{2} \mathrm{PO}_{4}$.

\section{MEASUREMENTS OF THE SEPARATE RELAXA- TION PROBABILITIES $P_{1}$ AND $P_{2}$}

In Fig. (8) the relaxation time is shown over an extended range of temperature with the crystal in the $\theta_{Z}=35^{\circ}$ orientation for which the $Y$ lines overlap. Between $220^{\circ}$ and $240^{\circ} \mathrm{K}, T_{1}$ and $T_{X Y}$ were found simultaneously by the procedure outlined in Sec. II. Above and below this temperature range, relaxation was found from the merged $Y$-line signal height obtained at a time of order $T_{1}$ after ending the saturation of this line. Above $240^{\circ} \mathrm{K}$, strong coupling between $X$ - and $Y$-spin systems exists, and consequently this procedure yielded $T_{1}=2\left(P_{X}+P_{Y}\right)^{-1}$. Below $220^{\circ} \mathrm{K}$ weak coupling exists, and the quantity measured was $T_{1 Y}=P_{Y}{ }^{-1}$.

The dashed lines in Fig. (8) represent the transition probabilities for three relaxation mechanisms which are postulated for the deuteron in this crystal, and the solid line is the sum of these transition probabilities. The component due to jumps between bonds is shown as calculated from the experimentally confirmed expressions as previously described.

The horizontal dashed line in Fig. (8) represents an estimate of the relaxation which is caused by spin diffusion to paramagnetic impurity ions. This estimate is based on proton and phosphorus relaxation times as measured by Jones ${ }^{8}$ in $\mathrm{KH}_{2} \mathrm{PO}_{4}$ and in the not completely deuterated $\mathrm{KD}_{2} \mathrm{PO}_{4}$ used in the present work. The diffusion-limited case ${ }^{9}$ is predicted, with a corresponding weak dependence of the relaxation on frequency and temperature. For a magnetic interaction between deuterons and the unpaired electron of a paramagnetic impurity ion, $\Delta m=2$ transitions do not occur.

${ }^{8}$ E. D. Jones (unpublished results).

${ }^{9}$ W. E. Blumberg, Phys. Rev. 119, 79 (1960). 
In the approximation that an effective value of transition probability can be used to describe this type of relaxation, the predicted probabilities are

$$
P_{1}=0.03 \mathrm{~min}^{-1}, \quad P_{2}=0 .
$$

Above $T_{c}$, the other relaxation mechanisms dominate to such an extent that more precise information on the spin diffusion mechanism is difficult to obtain. Below $T_{c}$, spin diffusion is probably the only important relaxation mechanism. A few experimental points to the right of the vertical line at $T_{c}$ in Fig. (8) suggest a rapid decrease in relaxation rate to the spin diffusion value for $T<T_{c}$ and other measurements which will be described later provide some additional evidence.

The third component of relaxation, obtained by subtracting the other two components from the total rate, is proportional to exp $(0.078 \mathrm{ev} / k T)$, is independent of magnetic field, and disappears rapidly as the temperature drops below the Curie point. It will be shown that this component can be described by EFG fluctuations due to jumps within the bond. Thus, it is described by the same Eqs. (34) and (35) of Appendix II as were used to describe relaxation due to jumps between bonds, except that the correlation time is different. The correlation time will be denoted by $T_{W}$. The condition $\omega T_{W} \ll 1$ must be assumed since the exponent in the dependence of the rate on temperature is positive. This condition is also consistent with the low value of the activation energy.

The hypothesis of random deuteron jumps within bonds which disappear rapidly below the Curie temperature is allowed by the neutron diffraction data of Bacon and $\mathrm{Pease}^{10}$ on $\mathrm{KH}_{2} \mathrm{PO}_{4}$ which show an elongated proton distribution above $T_{c}$ and a more concentrated distribution below $T_{c}$. This hypothesis is now put on a firmer basis by the relaxation measurements, and in particular, by measurements of $P_{1}$ and $P_{2}$ separately.

These measurements consisted of three sequences for disturbing and subsequently measuring population differences at temperatures for which $P_{X Y}$ is not of the same order of magnitude as $P_{1}$ and $P_{2}$. The corresponding equations for population differences during these sequences were solved for $P_{1}$ and $P_{2}$ with the redundant equation used to improve the accuracy.

One of these sequences is similar to that of Fig. (6) except that, immediately after ending the zero field period, the field was increased to its original value. The ratio of the subsequently measured signal height to the equilibrium value gives the time constant $T_{1}$ $=\left(P_{1}+2 P_{2}\right)^{-1}$ with which the spin system returns to equilibrium.

The other two sequences begin with the application of a saturating $\mathrm{rf}$ to one of the resonance lines. As shown in Fig. (9) the population difference of the pair of states corresponding to this line is quickly reduced to

${ }^{10}$ G. E. Bacon and R. S. Pease, Proc. Roy. Soc. (London) A230, 359 (1955).

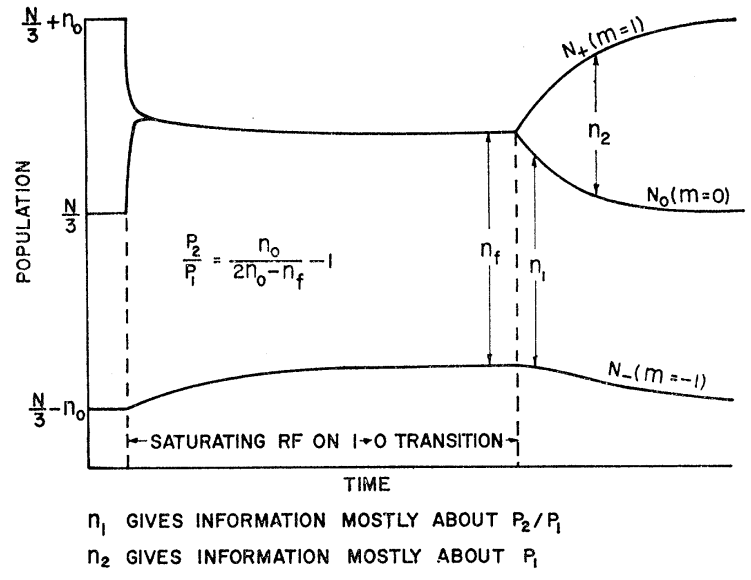

FIG. 9. Measurement methods for $P_{2} / P_{1}$ and $P_{1}$.

zero whereas the population difference of the other pair of states changes more slowly to an equilibrium value which is $\left[\left(P_{1}+2 P_{2}\right) /\left(P_{1}+P_{2}\right)\right] n_{0}$. The time constant is $\frac{2}{3}\left(P_{1}+P_{2}\right)^{-1}$. In one sequence the signal height corresponding to this population difference is measured as soon as possible after ending the saturation of the other line. The ratio of this signal height to the equilibrium value is used to determine $P_{2} / P_{1}$.

The third sequence is like the second except that, instead of measuring the signal height of the unsaturated line, the measurement is made on the saturated line at a time of order $T_{1}$ after ending saturation. Two time constants, $\left(3 P_{1}\right)^{-1}$ and $\left(P_{1}+2 P_{2}\right)^{-1}$, govern the approach to equilibrium. Since the coefficient of $\exp \left(-3 P_{1} t\right)$ is always the larger, this sequence provides information chiefly about $P_{1}$.

Values of $P_{1}$ and $P_{2}$ and their probable errors were found from the data obtained in the second and third measurement sequences using an IBM Model 650 computer. Because these sequences furnished information principally about $P_{1}$ and $P_{1} / P_{2}$, the percentage error for $P_{2}$ was larger than for $P_{1}$. The error in $P_{2}$ was then reduced considerably by combining the computer results with the value of $P_{1}+2 P_{2}$ obtained in the first measurement sequence when such results had been obtained. The results of these measurements and analysis at several temperatures and crystal orientations are shown in columns 4 and 6 of Table I.

The contribution to $P_{1}$ and $P_{2}$ due to deuteron jumps between symmetrically opposed positions on the bond axis will now be calculated. It will be shown that in the principal axis system of the static field gradient tensor the change in field gradient when the deuteron moves from one of these positions to the other has only two components, both nondiagonal. This is a consequence of crystal symmetry. This symmetry permits choice of atom pairs such that the perpendicular bisector of the line joining each pair is an axis parallel to the $\mathrm{Y}$ bonds which goes through the center of the $\mathrm{X}$ bond in question. Thus, for example, in the $c(Z)$ 
axis projection of the crystal structure shown in Fig. 10 one such pair for the $X_{+}$bond containing deuteron $\mathrm{D}_{+}$is composed of oxygens 1 and 2 which have coordinates $\left(-X_{0},-Y_{0}, Z_{0}\right)$ and $\left(X_{0},-Y_{0},-Z_{0}\right)$, respectively, relative to the bond center. The corresponding pair for the $X_{-}$bond containing deuteron $\mathrm{D}_{-}$are oxygens 3 and 4 which have coordinates relative to this bond center of $\left(-X_{0}, Y_{0},-Z_{0}\right)$ and $\left(X_{0}, Y_{0}, Z_{0}\right)$, respectively.

The changes in EFG seen by $\mathrm{D}_{+}$and $\mathrm{D}_{-}$in jumping along the bond axes from $(-S, 0,0)$ to $(S, 0,0)$ caused by like charges $n e$ at $\left(\mp X_{0},-Y_{0}, \pm Z_{0}\right)$ and $\left(\mp X_{0}, Y_{0}, \mp Z_{0}\right)$, respectively, have the nonvanishing components in the crystal system

$$
\begin{aligned}
& \Delta \phi_{X Y} \cong \pm \frac{12 n e Y_{0}}{R_{0}{ }^{5}}\left(1-\frac{5 X_{0}^{2}}{R_{0}^{2}}\right) S \equiv \pm A, \\
& \Delta \phi_{Y Z} \cong \frac{60 n e X_{0} Y_{0} Z_{0}}{R_{0}{ }^{7}} S \equiv B,
\end{aligned}
$$

in the approximation that $S=0.19 A^{\circ} \ll X_{0}$. The upper and lower signs are for $X_{+}$and $X_{-}$bonds, respectively, and $R_{0}^{2}=X_{0}^{2}+Y_{0}^{2}+Z_{0}^{2}$.

In order to calculate the relaxation rate, these changes in EFG components must be expressed in the laboratory system. One obtains for the $Z$ rotation $(Z$ and $z^{\prime}$ perpendicular, $H_{0}$ along $z^{\prime}, \theta_{Z}$ the angle between $X$ and $\left.z^{\prime}\right)$

$$
\begin{aligned}
& \Delta \phi_{x^{\prime} x^{\prime}}= \pm A \sin 2 \theta_{Z}, \\
& \Delta \phi_{y^{\prime} y^{\prime}}=0, \\
& \Delta \phi_{z^{\prime} z^{\prime}}=\mp A \sin 2 \theta_{Z}, \\
& \Delta \phi_{x^{\prime} y^{\prime}}=B \cos \theta_{Z}, \\
& \Delta \phi_{x^{\prime} z^{\prime}}= \pm A \cos 2 \theta_{Z}, \\
& \Delta \phi_{y^{\prime} z^{\prime}}=-B \sin \theta_{Z} .
\end{aligned}
$$

\begin{tabular}{|c|c|c|c|c|c|}
\hline \multirow[b]{2}{*}{$T\left({ }^{\circ} \mathrm{K}\right)$} & \multirow{2}{*}{$\begin{array}{l}\text { Orienta- } \\
\text { tion }\end{array}$} & \multicolumn{2}{|c|}{$P_{1}\left(\min ^{-1}\right)$} & \multicolumn{2}{|c|}{$P_{2}\left(\min ^{-1}\right)$} \\
\hline & & Calc & Meas & Calc & Meas \\
\hline 105 & $\theta_{Z}=90^{\circ}$ & 0.030 & $0.011 \pm 0.002^{\mathrm{a}}$ & 0 & $0.027 \pm 0.015^{\mathrm{a}}$ \\
\hline 205 & $=90^{\circ}$ & 0.030 & $0.032 \pm 0.004^{\mathrm{a}}$ & 0 & $0.016 \pm 0.009$ \\
\hline 210 & $=90^{\circ}$ & 0.130 & $0.130 \pm 0.011^{\mathrm{a}}$ & 0 & $0.036 \pm 0.017$ \\
\hline 215 & $=90^{\circ}$ & 0.120 & $0.102 \pm 0.008^{\mathrm{a}}$ & 0 & $0.001 \pm 0.003$ \\
\hline 215 & $=45^{\circ}$ & 0.060 & $0.054 \pm 0.003^{\mathrm{a}}$ & 0.075 & $0.094 \pm 0.022$ \\
\hline 215 & $=0^{\circ}$ & 0.060 & $0.050 \pm 0.006$ & 0.120 & $0.067 \pm 0.011$ \\
\hline 215 & $\theta_{X}=90^{\circ}$ & 0.090 & $0.082 \pm 0.008$ & 0.060 & $0.065 \pm 0.009$ \\
\hline 296 & $=90^{\circ}$ & 0.050 & $0.034 \pm 0.004$ & 0.029 & $0.049 \pm 0.003$ \\
\hline 343 & $=90^{\circ}$ & 0.043 & $0.043 \pm 0.003$ & 0.268 & $0.282 \pm 0.032$ \\
\hline 307 & $\theta_{Z}=0^{\circ}$ & 0.048 & $0.046 \pm 0.003$ & 0.023 & $0.021 \pm 0.003$ \\
\hline
\end{tabular}

In the $\theta_{X}=90^{\circ}$ orientation, the crystal and laboratory systems may be regarded as coincident, and the only nonvanishing components of the difference tensor are

$$
\begin{aligned}
& \Delta \phi_{x^{\prime} y^{\prime}}= \pm A, \\
& \Delta \phi_{y^{\prime} z^{\prime}}=B .
\end{aligned}
$$

TABLE I. Comparison of theory and experiment for $P_{1}$ and $P_{2}$ of $X$-bond deuterons.

a No $P_{1}+2 P_{2}$ data available to combine with computer results.

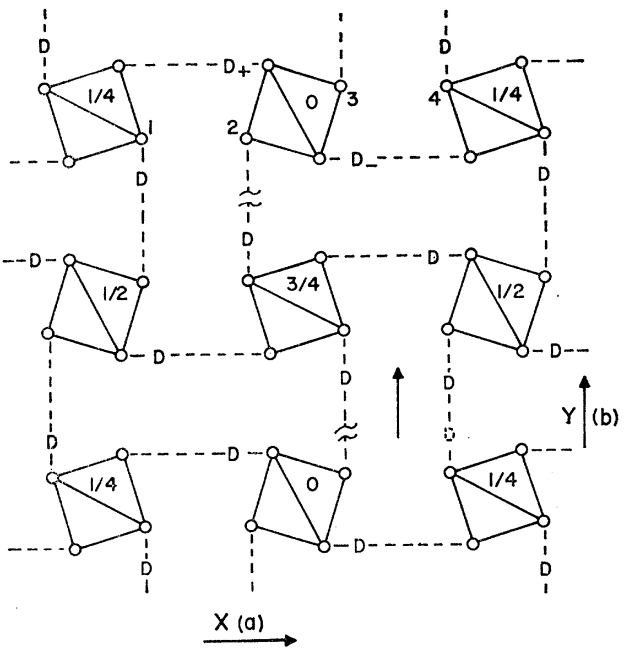

Fig. 10. $C(Z)$ axis projection of crystal structure.

Other orientations in $X$ rotation will not be required.

These are the field gradient components which are to be substituted into the equations

$$
\begin{aligned}
& P_{1}=\frac{1}{4} e^{2} Q^{2} T_{W} \hbar^{-2}\left[\left(\Delta \phi_{x^{\prime} z^{\prime}}\right)^{2}+\left(\Delta \phi_{y^{\prime} z^{\prime}}\right)^{2}\right], \\
& P_{2}=\frac{1}{8} e^{2} Q^{2} T_{W} \hbar^{-2}\left[\left(\Delta \phi_{x^{\prime} x^{\prime}}-\Delta \phi_{y^{\prime} y^{\prime}}\right)^{2}+4\left(\Delta \phi_{x^{\prime} y^{\prime}}\right)^{2}\right],
\end{aligned}
$$

obtained from Eqs. (34) and (35) of Appendix II by replacing $T_{X Y}$ by $T_{W}$ and using the condition $\omega T_{W} \ll 1$. Then for the $Z$ rotation

$$
\begin{aligned}
& P_{1}=2 C\left[A^{2} \cos ^{2} 2 \theta_{Z}+B^{2} \sin ^{2} \theta_{Z}\right], \\
& P_{2}=C\left[A^{2} \sin ^{2} 2 \theta_{Z}+4 B^{2} \cos ^{2} \theta_{Z}\right],
\end{aligned}
$$

where

$$
C=\frac{1}{8} e^{2} Q^{2} T_{W} \hbar^{-2} .
$$

Averaging over $X$ and $Y$ bonds $\left(P_{1}\right.$ and $P_{2}$ for $Y$ bonds are obtained by replacing $\theta_{Z}$ by $\left.\theta_{Z}+\frac{1}{2} \pi\right)$ gives

$$
\begin{aligned}
& \bar{P}_{1}=2 C\left[A^{2} \cos ^{2} 2 \theta_{Z}+\frac{1}{2} B^{2}\right], \\
& \bar{P}_{2}=C\left[A^{2} \sin 2 \theta_{Z}+2 B^{2}\right] .
\end{aligned}
$$

These averages must be used for $T$ greater than about $240^{\circ} \mathrm{K}\left(T_{X Y} \ll T_{1}\right)$; for $T$ less than about $220^{\circ} \mathrm{K}$ $\left(T_{X Y}>T_{1}\right)$ one should use the unaveraged quantities. Finally, for the orientation $\theta_{X}=90^{\circ}$, one obtains

$$
\begin{aligned}
& P_{1}=\bar{P}_{1}=2 B^{2} C, \\
& P_{2}=\bar{P}_{2}=4 A^{2} C .
\end{aligned}
$$

The experimental result at $215^{\circ} \mathrm{K}$ that in the $Z$ rotation $P_{1}$ is a maximum and $P_{2}$ is zero for $\theta_{Z}=90^{\circ}$ is predicted by Eqs. (12) and (13) independent of the values of $A$ and $B$ which are determined by the charges assigned to the various atom pairs. Other features of the $215^{\circ} \mathrm{K}$ orientation dependence can be fitted best by setting

$A^{2} C=0.015 \mathrm{~min}^{-1}, B^{2} C=0.030 \mathrm{~min}^{-1}$ at $215^{\circ} \mathrm{K}$. 
The ratio $B^{2} / A^{2}=2$ can be achieved by placing charges on the nearest two off-bond atom pair sites. Specifically, for $\mathrm{D}_{+}$in Fig. 10, charges ne at oxygens 1 and 2 and 1.1 ne at the nearest phosphorus atoms give this ratio.

An estimate of the correlation time $T_{W}$ can be obtained from the expression

$$
T_{W}{ }^{-1}=\nu_{W} \exp (-0.078 \mathrm{ev} / k T),
$$

with $\nu_{W} \sim k T / h$. At $T=215^{\circ} \mathrm{K}$ this gives $T_{W}=1.4 \times 10^{-11}$ sec. Through Eqs. (6), (7), and (19) one finds that this value of $T_{W}$ is consistent with a value of $n$ of order $\frac{1}{4}$ for the charges at the nearest two off-bond pairs of atoms described above. By comparison, charges of approximately $\frac{1}{2} e$ at the oxygen sites on the bond axis give the static EFG measured by $\mathrm{BU}$.

The measured values of $P_{1}$ and $P_{2}$ are compared in Table I with the sums of $P_{1}$ and $P_{2}$ for all three relaxation mechanisms. In computing these sums, averages over $X$ and $Y$ bonds are taken for $T>240^{\circ} \mathrm{K}$ and only $X$-bond values are used for $T<220^{\circ} \mathrm{K}$. The agreement is sufficiently good, both in magnitude and in angular dependence, to justify the hypothesis that the principal relaxation mechanisms over an extended range of temperatures above $T_{c}$ are in fact the three mechanisms which have been described. The agreement with relaxation time measurements reported by $\mathrm{BU}$ is also fairly good.

One additional consequence of jumps within bonds should be mentioned. Onsager ${ }^{11}$ has noted that the existence of rapid intrabond motion above the Curie point is indicated by the fast dielectric relaxation in $\mathrm{KH}_{2} \mathrm{PO}_{4}$ (no change in dielectric constant with frequency to at least $\left.2.5 \times 10^{10} \mathrm{cps}\right)^{12}$ and by the disappearance above $T_{c}$ of a small additional splitting of the deuteron lines observed by $\mathrm{BU}$, on the assumption that this splitting is due to a difference in EFG between the two possible deuteron sites within each bond. Subsequently, Uehling and Bjorkstam ${ }^{13}$ found that polarization of the crystal into a single domain removes this splitting, and noted that the splitting could be partially accounted for by the slight difference in bond directions in adjacent domains which are a consequence of the spontaneous shear.

The disappearance of the splitting for single domains is consistent with the existence of EFG differences between two sites in each bond of the type which have been discussed. As shown by $\mathrm{BU}, \phi_{z^{\prime} z^{\prime}}$ is the only EFG component that affects the resonant frequencies in first order. The change in $\phi_{z^{\prime} z^{\prime}}$ seen by an $X_{+}$bond deuteron in moving a distance $S$ to the right of the bond center is equal to the change seen by an $X_{-}$deuteron in moving an equal distance to the left [in Eqs. (8) the upper and lower signs are for $X_{+}$and $X_{-}$bonds, re-

${ }^{11} \mathrm{~L}$. Onsager (private communication).

${ }^{12}$ W. A. Yager, mentioned by W. P. Mason, reference 14, p. 258.

${ }^{13}$ E. A. Uehling and J. L. Bjorkstam, Bull. Am. Phys. Soc. 5, 345 (1960). spectively, and in addition the sign of $A$ depends on the sign of $S]$. Consequently, the $X_{+}$and $X_{-}$deuterons which are present in a single domain of the polarized crystal will have the same resonant frequencies since they will have moved to opposite ends of their respective bonds, as seen in Fig. 10.

\section{CONNECTION WITH ELECTRICAL CONDUCTIVITY}

Following a suggestion of Onsager ${ }^{11}$ that deuteron (or proton) jumping between bonds might govern electrical conductivity in $\mathrm{KH}_{2} \mathrm{PO}_{4}$ type crystals, measurements of conductivity as a function of temperature were made. Upon application of a potential difference, the current through the crystal rises quickly to a maximum value and then decays to an equilibrium value. Upon removal of the potential difference, the current reverses direction and then decays to zero. Measurements were made by running through this sequence repeatedly and recording the current. A potential difference of $200 \mathrm{v}$ was used.

The current decay was not strictly exponential, but had an effective time constant of the order of one minute as the conductivity varied by five orders of magnitude. This decay may be due to polarization effects at the electrodes. If this is the case, it is the initial conductivity which represents the true conductivity of the crystal. Both the initial and equilibrium conductivities are shown in Fig. 2.

The most extensive measurements were made on the same crystal (Clevite No. 3) as was used to obtain the values of $T_{X Y}$ and $T_{1}$ described earlier. These measurements provided an accurate value of the temperature dependence, but because of the irregular shape of this crystal they did not provide a good absolute determination of the conductivity. In order to obtain the latter, another crystal (Clevite No. 1) was used. The conductivity values for Clevite No. 3 which are recorded in Fig. 2 have been adjusted on this basis. The values are $\sigma=1.16 \times 10^{-10}(\mathrm{ohm} \mathrm{cm})^{-1}$ for the $c$-axis conductivity at $25^{\circ} \mathrm{C}$ with an activation energy of $0.582 \pm 0.020 \mathrm{ev}$. These values are of the same order of magnitude as obtained by $\mathrm{Mason}^{14}$ for $\mathrm{KH}_{2} \mathrm{PO}_{4}$ where the proton is responsible for the conductivity.

As shown in Fig. 2 the temperature dependence of the initial conductivity departs from exponential behavior near the Curie point. This may be due to lower jump barriers, as mentioned in Sec. II in connection with the anomalously low values of $T_{X Y}$ near $T_{c}$. Rapid fluctuations of the conductivity with time also occur at this temperature.

The most significant result is the strict proportionality within experimental error of $\left(T_{X Y} T\right)^{-1}$ and $\sigma$. Whether carriers (excess or vacancy deuterons) already in ex-

${ }^{14}$ W. P. Mason, Piezoelectric Crystals and their Applications to
Ultrasonics (D. Van Nostrand Company, Inc., New York, 1950), p. 140 . 
istence make jumps of length $\lambda$ over barriers of height $E_{j}$ or move freely with a mean free path $\lambda$, the relation between $P_{X Y}=\frac{1}{2} T_{X Y}{ }^{-1}$ and the diffusion constant $D$ is given by

$$
P_{X Y}=6 b g n D / N \lambda^{2},
$$

where $n$ and $N$ are the carrier and total deuteron concentrations respectively, $b$ is the probability that the moving deuteron changes identity in moving a distance $\lambda, g$ is the probability that an $X$ deuteron after becoming a carrier comes to rest in a $Y$ bond, and the numerical factor depends on the model. The factor 6 is appropriate for isotropic jumps through a fixed distance $\lambda$. The connection with $\sigma$ is now obtained by using the Einstein relation ${ }^{15} k T \mu=e D$ and writing $\sigma$ $=n e \mu$. One obtains

$$
P_{X Y}=6 b g k T \sigma /\left(N \lambda^{2} e^{2}\right) .
$$

In order to obtain the measured ratio of $P_{X Y}$ and $\sigma$, it is necessary to set $b g$ equal to 0.32 if $\lambda$ is to have its minimum reasonable value; namely, the mean deuteron separation $N^{-\frac{1}{3}}$, or $3.66 \AA$. A value somewhat greater than 0.5 would be expected for $g$ because the distances between the closest $X$ and $Y$ bonds are somewhat less than the smallest $X-X$ distances. Accordingly, $b$ must be somewhat less than 0.64 ; i.e., near unity, a result consistent with diffusion of vacant deuteron sites, and consistent also with diffusion of excess deuterons if it is assumed that the excess deuteron changes identity (replaces another deuteron from its bond) nearly every time it moves the interbond distance $N^{-\frac{1}{3}}$.

The results of the jump time and conductivity measurements are summarized in Eq. (4) and Fig. 2. The expression for $\sigma$ analogous to Eq. (4) for $T_{X Y}$ which has been used is

$$
\sigma=0.79 \exp (-0.58 \mathrm{ev} / k T) \text { in }(\mathrm{ohm} \mathrm{cm})^{-1} .
$$

Both $P_{X Y}$ and $\sigma$ are proportional to the product of carrier concentration $n$ and diffusion constant $D$. Attempts to separate the factors experimentally have so far been unsuccessful.

\section{CONNECTION WITH FERROELECTRICITY}

The existing theories of ferroelectricity in $\mathrm{KH}_{2} \mathrm{PO}_{4}$ type crystals are of two kinds :

(a) Theories which take account of long-range but not short-range interactions. The hydrogen bond is assumed to be symmetrical about its midpoint in the absence of polarization or external fields. Spontaneous polarization is a consequence essentially of a Boltzmann distribution over a few lowest lying energy levels.

(b) Order-disorder theories which neglect the long range forces but not the near-neighbor interactions. The role of the hydrogen bond is to contribute a proton to one or the other of the atomic groups con-

${ }^{15}$ A. Einstein, Ann. Physik 19, 371 (1906); J. Kirkwood, J. Chem. Phys. 14, 180 (1946).

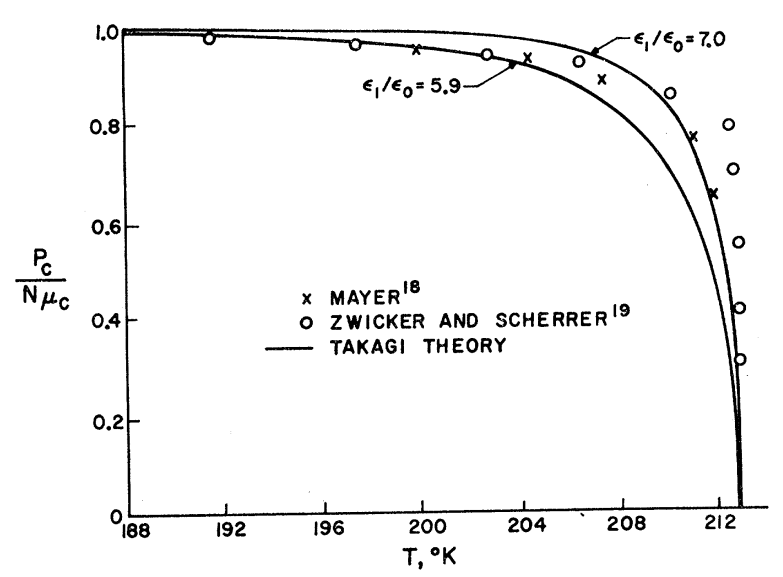

FIG. 11. Spontaneous polarization in $\mathrm{KD}_{2} \mathrm{PO}_{4}$.

nected by the bond. Such groups then possess internal energies which are determined by the arrangement of protons around it. Spontaneous polarization arises because the ordered or partially ordered state of low internal energy may also be a state of lower free energy than the completely disordered state.

The concepts involved in the second of these two types of theories lend themselves most naturally to the deuteron motions described in the earlier sections. A random intrabond motion with a correlation time $T_{W}$ which is strongly temperature dependent is consistent with these concepts, and the measured activation energy can be identified with one of the order-disorder energies. In particular, the measured activation energy of $0.078 \mathrm{ev}$ may be identified with the energy $\epsilon_{1}$ (ion pair energy) of the Takagi ${ }^{16}$ modification of the Slater ${ }^{17}$ theory, and the energy $\epsilon_{0}$ (energy of an unpolarized group above that of a polarized group) may be calculated from the Curie temperature and the measured value of $\epsilon_{1}$. Takagi shows that the temperature dependence of the spontaneous polarization depends on the ratio $\epsilon_{1} / \epsilon_{0}$. For $\mathrm{KD}_{2} \mathrm{PO}_{4}$ with $T_{c}=213^{\circ} \mathrm{K}$ and with $\epsilon_{1}=0.078 \mathrm{ev}$, one obtains $\epsilon_{0}=0.0132 \mathrm{ev}$ and $\epsilon_{1} / \epsilon_{0}=5$.9. The calculated polarization curve and a comparison with experimental data is shown in Fig. 11. ${ }^{18,19}$ Details of this calculation will be given in a separate publication.

\section{ACKNOWLEDGMENTS}

The authors gratefully acknowledge many helpful discussions with Professor Henry B. Silsbee and his guidance and assistance in setting up the computer program. We are indebted also to Wendland Beezhold for his assistance in some of the calculations.

\footnotetext{
${ }^{16}$ Y. Takagi, J. Phys. Soc. Japan 3, 271 and 273 (1948).

17 J. C. Slater, J. Chem. Phys. 9, 16 (1941).

18 R. J. Mayer, M. S. thesis, Department of Electrical Engineering, University of Washington, Seattle, Washington, 1961 (unpublished).

${ }_{19}$ B. Zwicker and P. Scherrer, Helv. Phys. Acta 17, 346 (1944).
} 
APPENDIX I. TIME CONSTANTS IN THE COUPLED SYSTEM OF $X$ - AND $Y$-BOND DEUTERONS

The $X$ - and $Y$-bond deuterons comprise a pair of spin systems coupled by the jumping of deuterons from one bond to the other. The important transition probabilities are $P_{1 X}$ and $P_{1 Y}$ for $\Delta m= \pm 1$ spin-lattice transitions in $X$ and $Y$ bonds respectively, $P_{2 X}$ and $P_{2 Y}$ for $\Delta m= \pm 2$ spin-lattice transitions, and $P_{X Y}$ for the jumping between bonds. Spin-spin transitions make no contribution to the change of populations of states except at orientations in which the frequency discrepancy is of the order of the linewidth, and are not included. Since there are three spin states, there are two independent variables describing the populations of states in each bond, and thus four independent variables for the coupled system. The differential equations describing the time rate of change of populations are obtained in the usual way. One obtains four coupled equations. For example, using the variables $x_{ \pm}$and $y_{ \pm}$ to represent the deviations of populations in the states $m= \pm 1$ of $X$ and $Y$ bonds, respectively, from their equilibrium values, the differential equations are

$$
\begin{aligned}
d x_{+} / d t=P_{1 X}\left(x_{0}-x_{+}\right)+ & P_{2 X}\left(x_{-}-x_{+}\right) \\
& +P_{X Y}\left(y_{+}-x_{+}\right),
\end{aligned}
$$

plus three other equations obtained from this one by the appropriate permutation or substitution of variables. Note that $x_{0}=-x_{+}-x_{-}$since the sum of the deviations is zero. Use has been made of the fact that the Zeeman splitting is small compared to $k T$ in order to simplify the equations. Also, use has been made of the fact that the additional quadrupole splitting $(\sim 100$ $\mathrm{kc} / \mathrm{sec}$ in frequency units) is small compared with the Zeeman splitting $(\sim 8 \mathrm{Mc} / \mathrm{sec})$. Note also that it is not assumed that $P_{1 X}=P_{1 Y}$ and $P_{2 X}=P_{2 Y}$ since it is shown in Sec. IV and Appendix II that these quantities are angular dependent and the orientations of $X$ and $Y$ bonds with respect to a magnetic field are different.

A partial decomposition of these coupled equations is obtained by a change of variables. Define

Then

$$
\begin{aligned}
& U_{ \pm}=x_{+} \pm x_{-}, \\
& V_{ \pm}=y_{+} \pm y_{-} .
\end{aligned}
$$

where

$$
\begin{aligned}
& d U_{+} / d t=-\left(P_{X Y}+3 P_{1 X}\right) U_{+}+P_{X Y} V_{+} \\
& d V_{+} / d t=P_{X Y} U_{+}-\left(P_{X Y}+3 P_{1 Y}\right) V_{+} \\
& d U_{-} / d t=-\left(P_{X Y}+P_{X}\right) U_{-}+P_{X Y} V_{-} \\
& d V_{-} / d t=P_{X Y} U_{-}-\left(P_{X Y}+P_{Y}\right) V_{-}
\end{aligned}
$$

$$
P_{X}=P_{1 X}+2 P_{2 X} \text { and } P_{Y}=P_{1 Y}+2 P_{2 Y} .
$$

The return of $U_{+}$and $V_{+}$to equilibrium is characterized by the time constants

$$
\begin{aligned}
&\left\{P_{X Y}+\frac{3}{2}\left(P_{1 X}+P_{1 Y}\right)\right. \\
&\left. \pm \frac{1}{2}\left[9\left(P_{1 X}-P_{1 Y}\right)^{2}+4 P_{X Y^{2}}\right]^{\frac{1}{2}}\right\}^{-1} .
\end{aligned}
$$

In the absence of coupling $\left(P_{X Y}=0\right), U_{+}$and $V_{+}$are governed, respectively, by the time constants $\left(3 P_{1 X}\right)^{-1}$ and $\left(3 P_{1 Y}\right)^{-1}$. Since $U_{+}=-x_{0}$ and $V_{+}=-y_{0}$, and since $x_{0}$ and $y_{0}$ are zero for a Boltzmann distribution, these time constants can be associated with the return of the spin systems to a Boltzmann distribution. For strong coupling $\left(P_{X Y} \gg P_{1 X}\right.$ and $\left.P_{1 Y}\right), U_{+}$and $V_{+}$are both governed by the time constants $\left[\frac{3}{2}\left(P_{1 X}+P_{1 Y}\right)\right]^{-1}$ and $\left(2 P_{X Y}\right)^{-1}$. The latter time constant will be defined as $T_{X Y}$, the time constant associated with the approach of the $X$ and $Y$ systems to equilibrium with each other.

The return of $U_{-}$and $V_{-}$to equilibrium is characterized by the time constants

$\left\{P_{X Y}+\frac{1}{2}\left(P_{X}+P_{Y}\right) \pm \frac{1}{2}\left[\left(P_{X}-P_{Y}\right)^{2}+4 P_{X X^{2}}\right]^{\frac{1}{1}}\right\}^{-1}$.

In the absence of coupling, $U_{-}$and $V_{-}$return to equilibrium with the time constants $P_{X}{ }^{-1}$ and $P_{Y}{ }^{-1}$, respectively. These time constants can be associated with the return to equilibrium of the system from a Boltzmann population distribution corresponding to a spin temperature different than the lattice temperature. For strong coupling, one time constant for both $U_{-}$and $V_{-}$is again $T_{X Y}$, while the other is $\left[\frac{1}{2}\left(P_{X}+P_{Y}\right)\right]^{-1}$. The latter time constant will be designated as the spinlattice relaxation time $T_{1}$. This designation is rather arbitrary, since there are, in general, four time constants governing the return of the spin system to equilibrium with the lattice.

The experimental information on populations is in the form of observed magnetic resonance signal heights, which are assumed proportional to differences in population of quantum states having adjacent $m$ values. These population differences are related to the previously described variables in the following manner:

$$
\begin{aligned}
& n_{X+}=x_{+}-x_{0}+n_{0}=\frac{3}{2} U_{+}+\frac{1}{2} U_{-}+n_{0}, \\
& n_{X-}=x_{0}-x_{-}+n_{0}=-\frac{3}{2} U_{+}+\frac{1}{2} U_{-}+n_{0},
\end{aligned}
$$

where $n_{0}$ is the equilibrium population difference at the lattice temperature. Similarly

$$
\begin{aligned}
& n_{Y_{+}}=\frac{3}{2} V_{+}+\frac{1}{2} V_{-}+n_{0}, \\
& n_{Y_{-}}=-\frac{3}{2} V_{+}+\frac{1}{2} V_{-}+n_{0} .
\end{aligned}
$$

Since general solutions for $U_{ \pm}$and $V_{ \pm}$are known, these equations provide general solutions for $n_{X_{ \pm}}$and $n_{Y_{ \pm}}$. The differential equations in $U_{ \pm}$and $V_{ \pm}$relate the arbitrary constants in $U_{+}$to those in $V_{+}$, and similarly for $U_{-}$and $V_{-}$. The initial conditions then fix the remaining four constants. Special applications of this procedure are given in Sec. II in connection with the measurement of $T_{X Y}$.

\section{APPENDIX II. CONTRIBUTION TO THE TRANSITION PROBABILITIES DUE TO JUMPING BETWEEN BONDS}

The transition probabilities due to random EFG fluctuations caused by deuteron jumps between $X$ and $Y$ bonds are calculated by a procedure similar to that 
TABLE II. Components of the EFG difference tensor.

\begin{tabular}{ccc}
\hline \hline$\Delta \phi_{i^{\prime} j^{\prime}}$ & $X$ rotation & $Z$ rotation \\
\hline$x^{\prime} x^{\prime}$ & $-\frac{1}{2} C\left(1-\cos 2 \theta_{X}\right)$ & $-C \cos 2 \theta_{Z}$ \\
$y^{\prime} y^{\prime}$ & $C$ & 0 \\
$z^{\prime} z^{\prime}$ & $-\frac{1}{2} C\left(1+\cos 2 \theta_{X}\right)$ & $C \cos 2 \theta_{Z}$ \\
$x^{\prime} z^{\prime}$ & $-\frac{1}{2} C \sin 2 \theta_{X}$ & $C \sin 2 \theta_{Z}$ \\
\hline
\end{tabular}

described in $\mathrm{BU}$ and used there to calculate the relaxation time $T_{1}$. It differs, however, in two important respects :

(a) It is not now assumed that the jumps between bonds are the only important cause of relaxation in this crystal.

(b) A specific motion responsible for the EFG fluctuations is postulated; namely, the jumps between bonds, and each of the relevant parameters which is needed in the calculation of spin transition probabilities, is assumed to be known; i.e., obtained from an independent measurement.

Consequently, the known jump time $T_{X Y}$ is used in place of the physically unspecified correlation time $\tau_{c}$ of $\mathrm{BU}$, and the differences of EFG components $\Delta \phi_{i j}$ between $X$ and $Y$ bonds, now assumed to be the amplitudes of the relevant random fluctuating variables, are used in place of the $\phi_{i j}$.

Then the transition probabilities for $\Delta m= \pm 1$ and $\Delta m= \pm 2$ transitions are, respectively,

$$
\begin{aligned}
& P_{1}=\frac{e^{2} Q^{2}\left[\left(\Delta \phi_{x^{\prime} z^{\prime}}\right)^{2}+\left(\Delta \phi_{y^{\prime} z^{\prime}}\right)^{2}\right]}{8 \hbar^{2}} \frac{2 T_{X Y}}{1+\left(\omega T_{X Y}\right)^{2}}, \\
& P_{2}=\frac{e^{2} Q^{2}\left[\left(\Delta \phi_{x^{\prime} x^{\prime}}-\Delta \phi_{y^{\prime} y^{\prime}}\right)^{2}+4\left(\Delta \phi_{x^{\prime} y^{\prime}}\right)^{2}\right]}{16 \hbar^{2}} \\
& \times \frac{2 T_{X Y}}{1+\left(2 \omega T_{X Y}\right)^{2}} .
\end{aligned}
$$

These expressions are identical with those given as Eqs. (8) and (9) in BU except for the changes already noted and the specialization to spin 1 . As before, primes
TABLE III. $P_{1}$ and $P_{2}$ due to deuteron jumps between bonds.

\begin{tabular}{ccc}
\hline $\begin{array}{c}\text { Transition } \\
\text { probability }\end{array}$ & $X$ rotation & $Z$ rotation \\
\hline$P_{1}$ & $\frac{\left(264.4 \times 10^{3}\right)^{2} \sin ^{2} 2 \theta_{X}}{36 \nu^{2} T_{X Y}}$ & $\frac{\left(264.4 \times 10^{3}\right)^{2} \sin ^{2} 2 \theta_{Z}}{9 \nu^{2} T_{X Y}}$ \\
& $\frac{\left(264.4 \times 10^{3}\right)^{2}\left(3-\cos 2 \theta_{X}\right)^{2}}{288 \nu^{2} T_{X Y}}$ & $\frac{\left(264.4 \times 10^{3}\right)^{2} \cos ^{2} 2 \theta_{Z}}{72 \nu^{2} T_{X Y}}$ \\
\hline
\end{tabular}

on coordinate components used as field gradient subscripts indicate that the field gradients are to be evaluated in the laboratory system with the magnetic field $H_{0}$ along the $z^{\prime}$ axis, and $\omega / 2 \pi$ is the $\Delta m= \pm 1$ transition frequency. Since $\omega T_{X Y} \gg 1$, even at the highest temperature and lowest magnetic field at which any of these measurements are made, the expressions may be simplified by writing $P_{1}$ and $P_{2}$ proportional to $\omega^{-2} T_{X Y}{ }^{-1}$.

The EFG components at the deuteron are given in BU. In units of $(2 h / 3 e Q) \times 10^{3} \mathrm{sec}^{-1}$, the values in the principal axis system are $179.2,-85.2$, and -94.0 along the bond axis, the perpendicular axis in the $X Y$ crystalline plane and the $Z$ axis, respectively. In this principal axis system, the change in EFG seen by a deuteron in going from a $Y$ - to an $X$-bond position can be described by an EFG difference tensor having nonzero components $\Delta \phi_{X X}=-\Delta \phi_{Y Y}=C$, where $C=(2 h / 3 e Q)\left(264.4 \times 10^{3}\right) \mathrm{sec}^{-1}$.

The values of the components in the laboratory system will now be tabulated for each of two perpendicular rotations of the crystal: (a) rotation about the crystalline $Z$ axis with $H_{0}$ perpendicular to the $Z$ axis and making an angle $\theta_{Z}$ with the crystalline $X$ axis; and, (b) rotation about the crystalline $X$ axis with $H_{0}$ perpendicular to the $X$ axis and making an angle $\theta_{X}$ with the crystalline $Y$ axis. The nonvanishing components are given in Table II.

Substituting into the expressions for $P_{1}$ and $P_{2}$, one obtains the results given Table III. 